\title{
Method for Segmentation of Knee Articular Cartilages Based on Contrast-Enhanced CT Images
}

\author{
Katariina A. H. Myller ${ }^{1,2}$ \\ Juuso T. J. Honkanen ${ }^{1,2,3}$ \\ Jukka S. Jurvelin ${ }^{1}$ \\ Simo Saarakkala ${ }^{4,5}$ \\ Juha Töyräs ${ }^{1,2}$ \\ Sami P. Väänänen ${ }^{1,2,6}$
}

1. Department of Applied Physics, University of Eastern Finland, Kuopio, Finland

2. Diagnostic Imaging Center, Kuopio University Hospital, Kuopio, Finland

3. Center of Oncology, Kuopio University Hospital, Kuopio, Finland

4. Research Unit of Medical Imaging, Physics and Technology, Faculty of Medicine, University of Oulu, Oulu, Finland

5. Department of Diagnostic Radiology, Oulu University Hospital, Oulu, Finland

6. Department of Orthopaedics, Traumatology and Hand Surgery, Kuopio University Hospital Kuopio, Finland 


\section{Abstract}

Segmentation of contrast-enhanced computed tomography (CECT) images enables quantitative evaluation of morphology of articular cartilage as well as the significance of the lesions. Unfortunately, automatic segmentation methods for CECT images are currently lacking. Here, we introduce a semiautomated technique to segment articular cartilage from in vivo CECT images of human knee. The segmented cartilage geometries of nine knee joints, imaged using a clinical CTscanner with an intra-articular contrast agent, were compared with manual segmentations from CT and magnetic resonance (MR) images. The Dice similarity coefficients (DSCs) between semiautomatic and manual CT segmentations were $0.79-0.83$ and sensitivity and specificity values were also high (0.76-0.86). When comparing semiautomatic and manual CT segmentations, mean cartilage thicknesses agreed well (intraclass correlation coefficient $=0.85-0.93$ ); the difference in thickness (mean $\pm \mathrm{SD}$ ) was $0.27 \pm 0.03 \mathrm{~mm}$. Differences in DSC, when MR segmentations were compared with manual and semiautomated CT segmentations, were statistically insignificant. Similarly, differences in volume were not statistically significant between manual and semiautomatic CT segmentations. Semiautomation decreased the segmentation time from $450 \pm 190$ to $42 \pm 10 \mathrm{~min}$ per joint. The results reveal that the proposed technique is fast and reliable for segmentation of cartilage. Importantly, this is the first study presenting semiautomated segmentation of cartilage from CECT images of human knee joint with minimal user interaction.

\section{Keywords}

Automation, Contrast Agent, Knee Joint, Musculoskeletal Imaging 


\section{Introduction}

Osteoarthritis (OA) is a common cause of immobility and pain, thus inducing major socioeconomic burden.16 The pathological changes in articular cartilage, subchondral bone, and synovial fluid, induced by injury or trauma, may lead to advanced OA.3 The early signs of post-traumatic OA include disruption of cartilage collagen network, depletion of glycosaminoglycans (GAGs), and alterations in the structure of subchondral bone and the thickness of the subchondral bone plate. 4 These alterations should be observed early in order to prevent the progression to post-traumatic OA.11,27 Unfortunately, the alterations are usually diagnosed at a stage when little remains to prevent the progress of OA or repair the initial changes.

A recent study suggested that delayed computed tomography arthrography (dCTa) imaging could permit simultaneous detection of changes in articular cartilage and subchondral bone quantitatively in vivo.26 In $\mathrm{dCTa}$, contrast agent solution is injected into the joint capsule enabling its diffusion into articular cartilage. The diffusion characteristics make it possible to determine the integrity of cartilage; i.e., anionic contrast agent diffuses into cartilage inversely proportionally to GAG content,24,25,35 and the diffusion is also dependent on the integrity of the collagen network as well as the water content of cartilage.14,18 Previously, arthrographic contrast-enhanced computed tomography (CECT) images, i.e., images that are taken immediately after contrast agent administration, have also been confirmed to detect cartilage lesions 14 that are common in joint injuries related to post-traumatic OA. Bone mineral density of subchondral bone can also be determined with CT,26,42 provided that the hydroxyapatite phantoms are set around the joint for density calibration. In contrast to magnetic resonance imaging (MRI), CECT enables simultaneous visualization of bone and cartilage with high signal-to-noise ratio and resolution.

To exploit quantitative dCTa analysis to simultaneously study the morphology and composition of articular cartilage, the accurate segmentation of tissues is a prerequisite. Conventionally, segmentation has been conducted manually and, therefore, it has been extremely time-consuming, decreasing the clinical feasibility of dCTa. An automated segmentation method would be a significant advance since it could enable a quantitative analysis of dCTa images in the clinical setting, i.e., if it were included as a part of a routine examination, it would improve the diagnostics of knee injuries. In particular, the accurate and reproducible evaluation of focal cartilage defects and analysis of alterations in cartilage thickness would help to detect initial OA-related changes and acute cartilage injuries.7 Furthermore, biomechanical modelling of the knee joint requires segmentation of the joint 
structures, 46 and arthrographic CECT images may be utilized as an input. However, the clinical applicability of such techniques suffers critically due to lack of automated segmentation.

Several semiautomatic or automatic segmentation techniques, based, for instance, on statistical modeling,50 active shape modeling,10,30 texture recognition,34 and atlases,28 have been developed to segment bones and cartilage from MR images. Tabrizi et al. have introduced a method to segment acetabular cartilage from CECT images38 and automatic methods exist to segment bone from CT images.4, 5, 6, 30 Nonetheless, automated methods capable of segmenting cartilage from arthrographic CECT images of human knee joint are lacking.

In this study, we introduce a semiautomated technique to segment articular cartilage and subchondral bone from in vivo arthrographic CECT images of knee joint, i.e., from distal femur and proximal tibia. The accuracy and feasibility of semiautomatic segmentation are evaluated by comparing semiautomatic CT segmentations with manual segmentations based on CT and MR images. The aim of the present method is to significantly reduce the time needed for segmentation, to minimize user interaction, and concurrently achieve an accuracy similar to manual segmentation.

\section{Materials and Methods}

Patients ( $n=9,2$ males and 7 females, age 50-68) with persistent knee pain were enrolled in the study after a clinical examination. All these patients had diagnosed cartilage lesions and/or meniscal tears, rupture or injury, likely caused by rotational trauma.36 The CECT imaging of the patients' knees was approved by the Ethical Committee of the Northern Ostrobothnia Hospital District (Decision No. $33 / 2010)$.

Prior to the imaging, an anionic contrast agent, $20 \mathrm{~mL}$ of ioxaglate $\left(105 \mathrm{mM}\right.$, Hexabrix ${ }^{\mathrm{TM}} 320$, Guerbet, Roissy, France) diluted in $0.9 \%$ saline, was injected into the knee joint capsule. The patients flexed and extended their legs for 5 min ensuring an even distribution of the contrast agent in the joint space. Subsequently, the knee was imaged using a clinical CT-scanner (Discovery ${ }^{\mathrm{TM}}$, PET/CT, 690 GE Medical Systems, Waukesha, WI, USA) with a tube voltage of $100 \mathrm{kV}$, the pitch of 0.53 , and a voxel size of $0.3 \times 0.3 \times 0.3 \mathrm{~mm}^{3}$. The imaging time was less than $30 \mathrm{~s}$ and the built-in filtering of the system corresponded to $4.3 \mathrm{~mm}$ of aluminium.

As a reference, the same knees were MR imaged within 2 weeks of CECT imaging. MRI of knees was conducted using a 3 T scanner (Siemens Skyra, Siemens Healthcare, Erlangen, Germany) with a 
15-channel transmit/receive knee coil (Quality Electrodynamics (QED), Mayfield Village, OH, USA). A double echo steady state sequence with water excitation (repetition time/time to echo $=14.1 / 5 \mathrm{~ms}$ ) was performed to image the anatomy of the knee. The field of view was $150 \times 150 \mathrm{~mm}^{2}$, matrix size $256 \times 256$, and slice thickness $0.6 \mathrm{~mm}$, resulting in an isotropic voxel size of $0.6 \times 0.6 \times 0.6 \mathrm{~mm}^{3}$.

The overview of the semiautomatic segmentation process is presented in Fig. 1 and in this section. A more detailed explanation of the segmentation steps is presented in the following sections. First, the surfaces of distal femur and proximal tibia were generated from arthrographic CECT images using Stradwin (v. 5.2, Department of Engineering, University of Cambridge, UK). Thereafter, the outer surfaces of the bones, i.e., periosteal surfaces, were segmented using Stradwin and, furthermore, the outcome was corrected using a custom-made smoothing (MATLAB, R2015a, MathWorks, Inc., Natick, MA, USA). A template periosteal surface mesh, from a previous study,46 was registered nonrigidly to the patient's periosteal surface using MATLAB, collating information about the anatomical location of articular cartilage, i.e., the bone-cartilage interface, to the mesh. Intensity profiles were captured along surface normals for each vertex point at the region of cartilage. Contrast-enhancement of joint space enabled the localization of the articular surface from the intensity profile; the surface was detected from the intensity profiles using a half-maximum-based algorithm written in MATLAB. The details of this procedure are explained later in the text. 


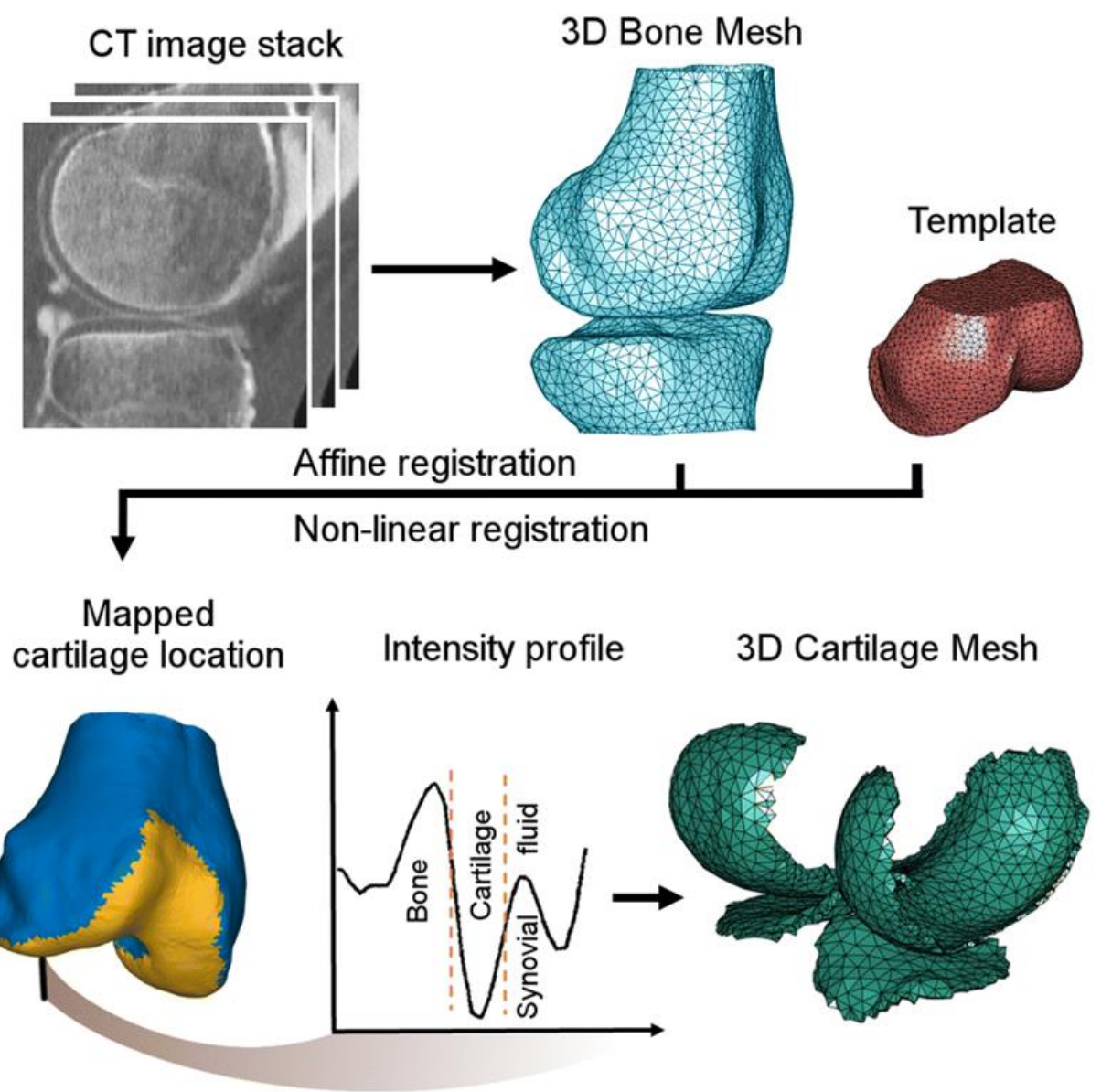

Figure 1. Overview of the segmentation. Periosteal surfaces of the patient's bone were generated using Stradwin software. In MATLAB, these meshes were first affinely and thereafter nonlinearly registered to the template mesh in order to localize cartilage anatomically. From the intensity profiles, generated along the surface normals, the cartilage surface was defined as a half-maximum of local minimum (middle of cartilage) and maximum (synovial fluid, i.e., contrast agent). The 3D cartilage mesh was constructed from these points. 
First, the bone surface was segmented semiautomatically. The 3D meshes for distal femur and proximal tibia were generated using Stradwin software. In the initial manual step, 10-15 axial contours per bone were manually drawn on bone cortex. Thereafter, the initial bone surface was generated from these sparse cross sections using region correspondence.41 This manual step took $16 \pm 4$ min (mean $\pm \mathrm{SD}$ ) per bone whereas the latter bone segmentation steps were automatic. Stradwin creates a periosteal surface, i.e., the outer surface of cortical bone, based on the intensity profiles generated perpendicular to the semiautomatically generated initial bone surface.41 For each initial bone mesh, three periosteal surface meshes were generated using different intensity profile lengths (femur: 6,8 and $10 \mathrm{~mm}$, tibia: 8,10 , and $12 \mathrm{~mm}$ ) and averaging each profile with the width of three pixels $(1.2 \mathrm{~mm})$. This was done to correct local inaccuracies on the bone surface; the best fit for the bone surface was defined subsequently in MATLAB. Each vertex point was analysed separately. At each point, the best fitting surface profile was selected based on the mutual correspondence of the surfaces and the internal confidence parameter calculated by Stradwin.41 In the final smoothing step, a vertex was defined as unsmooth if the two neighbouring vertices disagreed by more than $5 \mathrm{~mm}$ in the direction of surface normal, i.e., there was a clear error in the preceding segmentation. These, $0-32(<0.1 \%$ of total) points per bone, were further smoothed by setting them to the average of their neighbouring vertices to eliminate any spikes and achieve smooth periosteal surface. The bone surfaces were assumed to be intact or, at worst, only moderately affected by OA and hence smoothing could be conducted reliably.

After bone segmentation, articular cartilage surfaces were segmented. Cartilage regions were determined by registering the template bone mesh to the periosteal surface mesh of patient's bones, gathering information about the anatomy of cartilage to the mesh (Fig. 1). First, the template mesh was mirrored in case being in the contralateral side compared with the patient's periosteal surface mesh. Correspondence between the template bones and patient's bones was defined by setting manually 15 landmarks on the femur and 12 on the tibia on the periphery of tibial and femoral cartilages and on the metaphysis of bone (Supplementary material, Fig. 1). Typically, positioning of these landmarks took $5 \pm 2 \min (\operatorname{mean} \pm \mathrm{SD})$ per bone; the rest of the steps were automatic. The landmarks defined a geometric displacement field, generated with thin-plate splines algorithm,2,43,44 between the template bones and the patient's bones. This thin-plate spline field was used to warp the template bone to the shape of patient's bone. The periosteal surfaces of template bones were registered affinely on the patient's bones using an iterative closest point algorithm.17 In the final registration step, a non-rigid mesh registration algorithm 21 with seven iteration rounds was used, decreasing the distance between the meshes to less than $0.1 \mathrm{~mm}$. Finally, the closest 
counterpartying vertices between the template and patient's periosteal mesh were determined, thereby bringing anatomical localization from the template to the patient's surface mesh.

The numbers of cartilage vertices (mean \pm SD) were $3017 \pm 1054$ and $1127 \pm 312$ for femoral cartilage and tibial cartilage, respectively, depending on the patient-specific shape and size of the knee. Similarly as in the determination of cortical bone,41 intensity profiles were captured along surface normals at the region of cartilage in MATLAB. Ten-millimetre-long intensity profiles were captured, averaged orthogonally to the generated normal line with a diameter of 3 pixels, and sampled with $0.1 \mathrm{~mm}$ intervals resulting in 100 samples (Supplementary material, Fig. 2). The cartilage surface was defined from the intensity profiles as a half-maximum at local minimum (middle of cartilage) and maximum (contrast agent in the joint space) (Fig. 2). First, the custom-made algorithm searched all local minima and maxima from the intensity profile and selected those nearest to bone. If the difference in Hounsfield unit (HU) values between the selected extremes (minimum and maximum) were less than the threshold value of $50 \mathrm{HU}$, these minimum and maximum values were ignored and the sequential minimum and maximum were selected from the intensity profile. The searching was continued until the threshold was exceeded. Based on the selected maximum and minimum, the mean HU value was defined and its location on the profile, i.e., the location of the halfmaximum, was determined. These half-maximum points formed vertices of the cartilage surface mesh. 


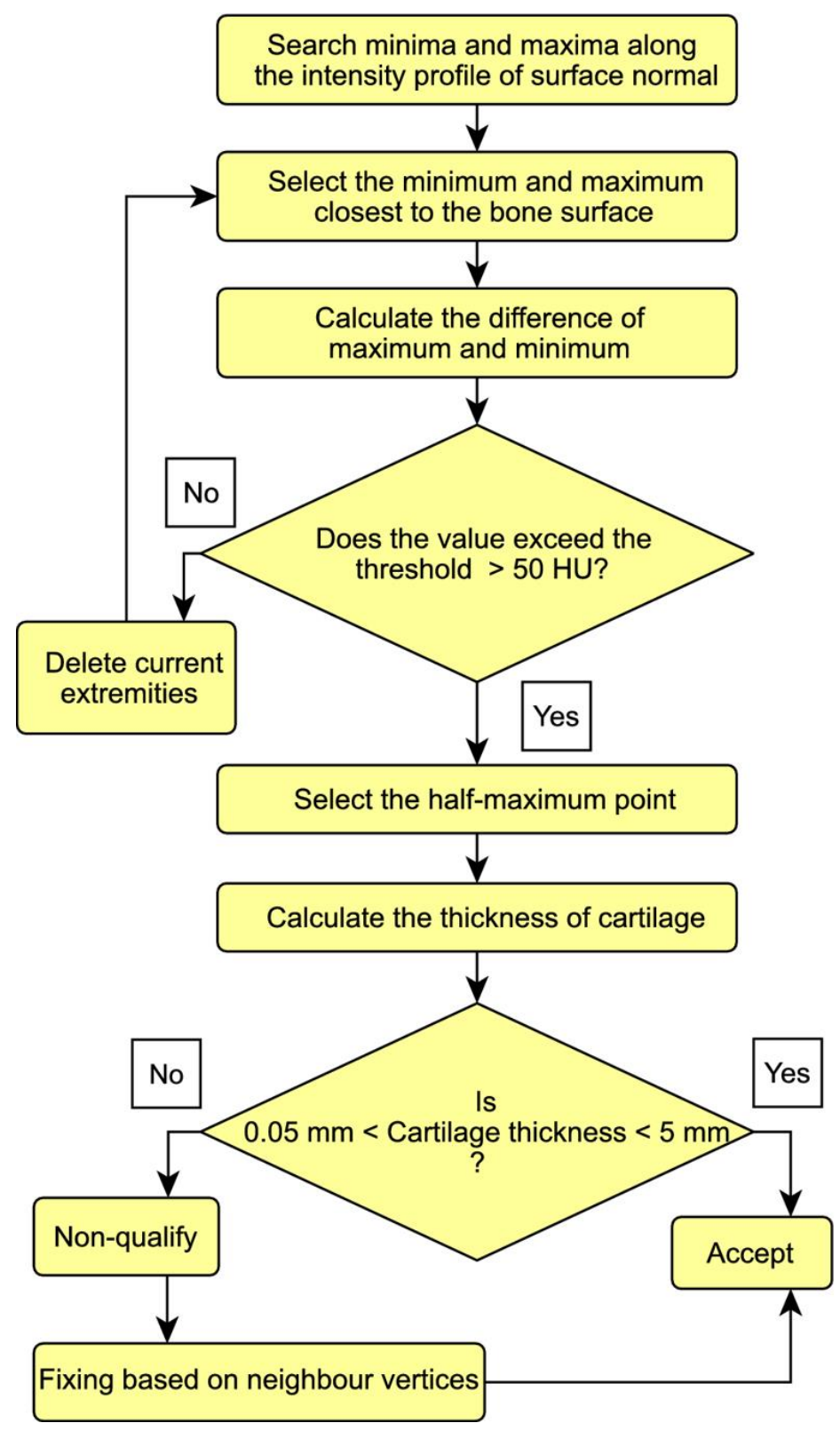

Figure 2. Schematic diagram of the modified half-maximum algorithm for cartilage surface detection from contrast-enhanced images. 
The cartilage surface was modified afterwards. The cartilage vertex point was determined to be erroneous if the determined cartilage thickness was 50\% more than the mean of the neighbouring vertices, higher than $5 \mathrm{~mm}$ or less than $0.05 \mathrm{~mm}$. These clearly faulty points were replaced by the median of the non-erroneous neighbour vertices. The thickness was set to $0.5 \mathrm{~mm}$ in the outermost rim of the cartilage based on a previous study.13 Furthermore, to serve also future FE modelling purposes, thickness was set to be greater than one pixel size. This rim was excluded from the analysis of the thickness results. In the final step, the final 3D cartilage surface mesh enclosing the cartilage volume inside was formed by triangulating the cartilage rim between the cartilage and periosteal surface meshes.

As a reference, cartilages were segmented also manually (Seg3D, v2.2.1, University of Utah, UT, USA) by two of the authors (KAHM and JTJH) from CT images prior to semiautomatic segmentation. Surface meshes were generated from the manual segmentation masks using Mimics software (Materialise, v15.0, Leuven, Belgium) and, thereafter, analysed with MATLAB. Moreover, cartilages were manually segmented (KAHM) from the MR images using the same protocol as in the manual CT segmentations. When studying the impact of the user interaction, i.e., intra-user and inter-user agreement of semiautomatic segmentations, manual tasks of semiautomatic segmentation were conducted (twice by KAHM and once by JTJH) and compared. The volume and thickness of cartilage were determined to evaluate the feasibility of the method to measure these clinically important parameters.8,12,29 The cartilage volume was calculated from surface meshes. Thickness values of semiautomatically generated cartilage meshes were determined as the length of the normals between bone-cartilage interface and cartilage surface, i.e., distance between vertices. The thickness values of manually segmented cartilage geometries were calculated as the shortest distance between the nodes on periosteal and cartilage surfaces (Euclidean distance). Spatial variation of cartilage thickness was visualized as $2 \mathrm{D}$ projected surfaces. Thus, prior to this step, the femoral cartilages were fitted to a cylinder shape and then projected to a $2 \mathrm{D}$ plane. 8

Manual and semiautomatic segmentations were compared using the Dice similarity coefficient (DSC) which has been postulated to be suitable when comparing spatial correspondences.51 DSC was calculated as

$$
D S C=\frac{2 J}{1+J},
$$


where $J$ is the Jaccard index calculated as

$$
J=\frac{V_{\text {inter }}}{V_{\text {union }}}
$$

where $V_{\text {inter }}$ is the intersection between segmentations, and $V_{\text {union }}$ is the union between segmentations. In order to compare segmentations comprehensively, specificity and sensitivity were calculated as

$$
\text { sensitivity }=\frac{V_{\text {inter }}}{V_{\text {manual }}}
$$

and

$$
\text { specificity }=1-\frac{V_{\text {semiauto }}-V_{\text {inter }}}{V_{\text {manual }}} .
$$

Similarly to the volumes, the intersections and unions were calculated from the surface meshes. MR and CT images had different coordinate systems and, hence, prior to the analysis, surface meshes determined from MR images were co-registered with the surface meshes determined from CT images using an open-source software wxRegSurf (v. 16, Department of Engineering, University of Cambridge, UK). The significance of differences was studied using Friedman test (IBM ${ }^{\circledR}$ SPSS $^{\circledR}$ Statistics, v. 21, 2012, Armonk, NY, USA). Mean thickness values of cartilage were compared using a reproducibility coefficient alongside Bland-Altman visualization. Intraclass correlation coefficient (ICC) was used to study the statistical repeatability of the segmentation methods (IBM ${ }^{\circledR}$ SPSS $^{\circledR}$ Statistics).

\section{Results}

The values of $D S C$, sensitivity and specificity between all the semiautomatic segmentations were very similar (Table 1) and no significant difference was found in these three parameters between semiautomatic segmentations. Furthermore, ICC values showed a good correspondence between the segmentations (Table 1). Since the correspondence was similar between all three semiautomatic segmentation rounds, one was randomly selected to be compared with the manual segmentations. 
Table 1. Dice similarity coefficient $(D S C)$, sensitivity, specificity (mean $\pm \mathrm{SD}$ ), and intraclass correlation coefficients for volume and thickness between three semiautomatic segmentations of CT images by two authors.

\begin{tabular}{lllllll}
\hline & \multicolumn{5}{c}{ Femur } & \multicolumn{5}{c}{ Tibia } \\
\cline { 2 - 6 } & DSC & Sensitivity & Specificity & DSC & Sensitivity & Specificity \\
\hline \hline A1 vs. A2 & $0.83 \pm 0.04$ & $0.85 \pm 0.04$ & $0.79 \pm 0.07$ & $0.78 \pm 0.04$ & $0.80 \pm 0.04$ & $0.75 \pm 0.09$ \\
A1 vs. B1 & $0.82 \pm 0.05$ & $0.85 \pm 0.04$ & $0.77 \pm 0.07$ & $0.78 \pm 0.03$ & $0.81 \pm 0.05$ & $0.73 \pm 0.10$ \\
A2 vs. B1 & $0.82 \pm 0.04$ & $0.83 \pm 0.04$ & $0.81 \pm 0.05$ & $0.78 \pm 0.04$ & $0.79 \pm 0.07$ & $0.76 \pm 0.10$ \\
\hline
\end{tabular}

Intraclass correlation coefficient $[\mathrm{CI}]$

\begin{tabular}{lllll}
\hline \hline & A1 vs. A2 & A1 vs. B1 & A2 vs. B1 & A1 vs. A2 vs. B1 \\
Volume & $0.98[0.940 .99]^{*}$ & $0.97[0.910 .99]^{*}$ & $0.97[0.971 .00]^{*}$ & $0.98[0.950 .99]^{*}$ \\
Thickness & $0.92[0.810 .97]^{*}$ & $0.83[0.600 .93]^{*}$ & $0.84[0.620 .94]^{*}$ & $0.86[0.730 .94]^{*}$ \\
\hline
\end{tabular}

$A$ author A, $B$ author B, 1 first segmentation, 2 second segmentation, $C I$ confidence interval

*Statistical significance $p \leq 0.001$ 
Semiautomatic segmentations (Semiautomatic) and two manual segmentations (Manual 1 and Manual 2), determined from CT images, were compared with each other. The agreements were high between semiautomatic and two manual segmentations, as measured with DSC (0.79-0.83), sensitivity (0.78-0.84) and specificity (0.76-0.86), which is also shown in the representative 3D image of femoral segmentations (Supplementary material, Fig. 3). The agreements were slightly higher in femoral than in tibial cartilage. The best agreement of sensitivity in femoral cartilage was found between the semiautomatic segmentation and Manual $1(0.84)$ but the overall correspondence of all of the parameters was slightly better between the manual segmentations than between the semiautomatic and manual ones (Table 2). 
Table 2. Segmentation parameters calculated from segmentations of CT images: Dice similarity coefficient, sensitivity, and specificity for femoral and tibial cartilages.

\begin{tabular}{|c|c|c|c|}
\hline \multicolumn{2}{|r|}{ FEMUR } & MEAN & SD \\
\hline \multirow{3}{*}{$\begin{array}{c}\text { Dice } \\
\text { Similarity } \\
\text { Coefficient }\end{array}$} & Semiautomatic vs. Manual 1 & 0.83 & 0.03 \\
\hline & Semiautomatic vs. Manual 2 & 0.81 & $0.03 \cdots$ \\
\hline & Manual 1 vs. Manual 2 & 0.86 & $0.02 \ldots$ \\
\hline \multirow{3}{*}{ Sensitivity } & Semiautomatic vs. Manual 1 & 0.84 & 0.04 \\
\hline & Semiautomatic vs. Manual 2 & 0.78 & $0.04=$ \\
\hline & Manual 1 vs. Manual 2 & 0.82 & i \\
\hline \multirow{3}{*}{ Specificity } & Semiautomatic vs. Manual 1 & 0.81 & 0.05 \\
\hline & Semiautomatic vs. Manual 2 & 0.86 & 0.04 \\
\hline & Manual 1 vs. Manual 2 & 0.92 & 0.03 \\
\hline \multicolumn{2}{|r|}{ TIBIA } & MEAN & SD \\
\hline \multirow{3}{*}{$\begin{array}{c}\text { Dice } \\
\text { Similarity } \\
\text { Coefficient }\end{array}$} & Semiautomatic vs. Manual 1 & 0.79 & $0.03^{--}$ \\
\hline & Semiautomatic vs. Manual 2 & 0.79 & $0.03 \cdots$ \\
\hline & Manual 1 vs. Manual 2 & 0.87 & 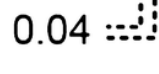 \\
\hline \multirow{3}{*}{ Sensitivity } & Semiautomatic vs. Manual 1 & 0.82 & 0.04 \\
\hline & Semiautomatic vs. Manual 2 & 0.79 & 0.04 \\
\hline & Manual 1 vs. Manual 2 & 0.85 & 0.06 \\
\hline \multirow{3}{*}{ Specificity } & Semiautomatic vs. Manual 1 & 0.76 & $0.09 \cdots$ \\
\hline & Semiautomatic vs. Manual 2 & 0.79 & 0.06 \\
\hline & Manual 1 vs. Manual 2 & 0.90 & 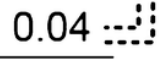 \\
\hline
\end{tabular}

$\cdots$ Statistically (Friedman's test) significantly different $(p<0.05)$

values are connected with a dashed line. 
The agreement in cartilage mean thickness (Fig. 3) determined from semiautomatic and manual segmentations was high; reproducibility coefficients $(0.15-0.18 \mathrm{~mm})$ were very similar for all the comparisons. The mean absolute differences between the thickness values were 0.25 and $0.31 \mathrm{~mm}$ for femoral cartilage and 0.26 and $0.27 \mathrm{~mm}$ for tibial cartilage (Semiautomatic vs. Manual 1, and Semiautomatic vs. Manual 2, respectively). Cartilage thickness maps revealed that the agreement was best in the middle of articular surface and slightly lower in the in regions close to the joint periphery (Fig. 4). ICCs between femoral cartilage thickness values, determined using manual (Manual 1 and Manual 2) and semiautomatic segmentations, were 0.93 [95\% confidence interval $(\mathrm{CI})=0.71-0.98$ ] and $0.85(95 \% \mathrm{CI}=0.47-0.96)$, respectively. When comparing tibial cartilage thicknesses, ICC was $0.93(95 \% \mathrm{CI}=0.73-0.89)$ between semiautomatic and Manual 1 segmentations, and $0.93(95 \%$ $\mathrm{CI}=0.71-0.98)$ between semiautomatic and Manual 2 segmentations. 
Semiautomatic vs. Manual 1

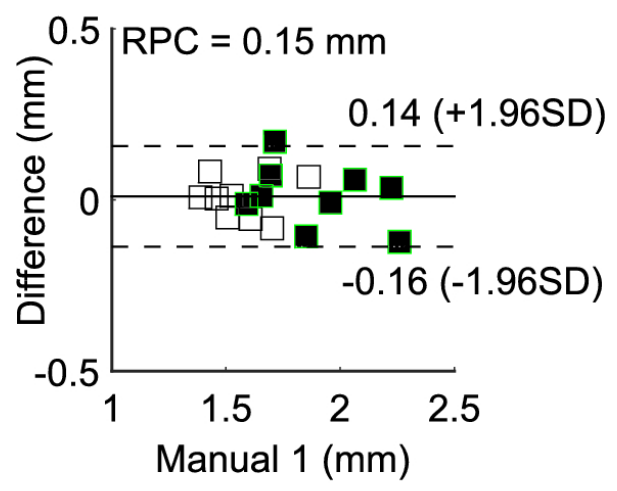

Semiautomatic vs. Manual 2

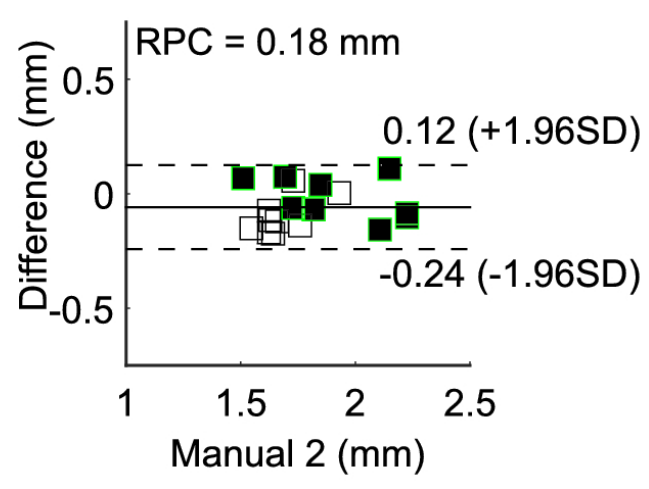

Manual 1 vs. Manual 2

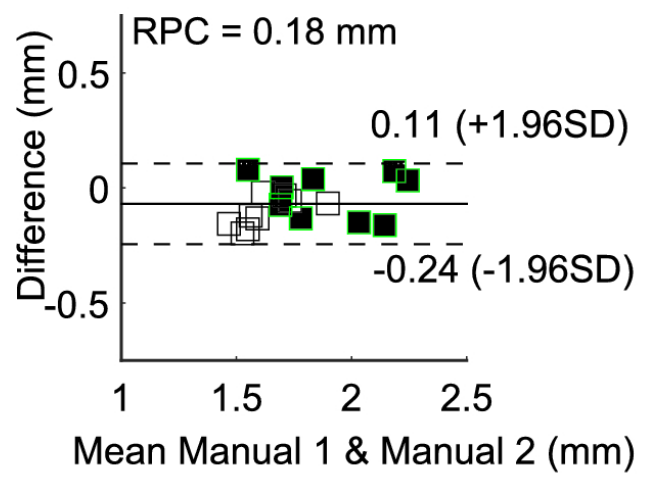

femoral cartilage $\mathbf{a}$ tibial cartilage

$\mathrm{RPC}=$ reproducibility coefficient

Figure 3. Mean values of cartilage thickness; manual segmentations (Manual 1 and Manual 2) compared with the semiautomatic segmentations (Semiautomatic) and manual segmentations compared with each other. The Bland-Altman plots show that the segmentation techniques agree well. 

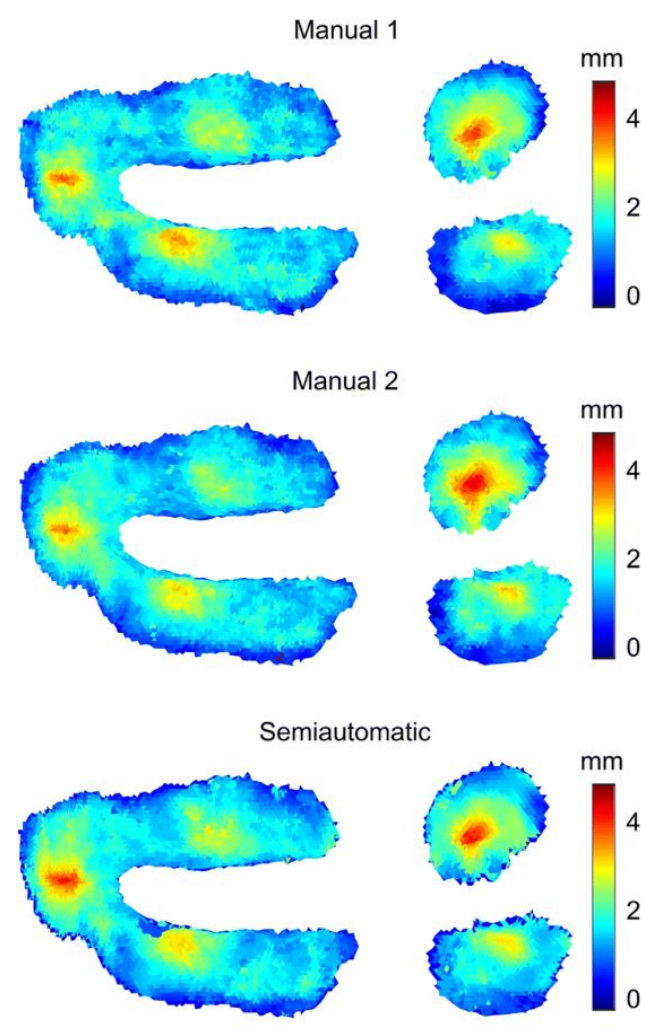

Difference

Manual 1 - Manual 2

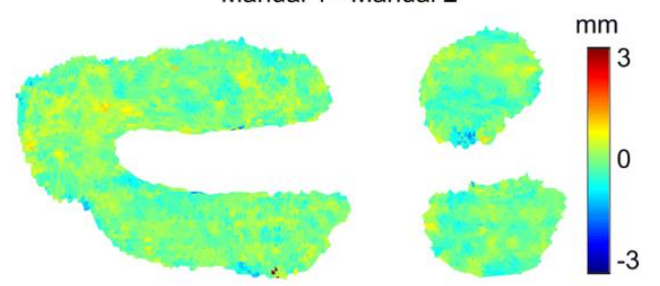

Difference

Manual 1 - Semiautomatic

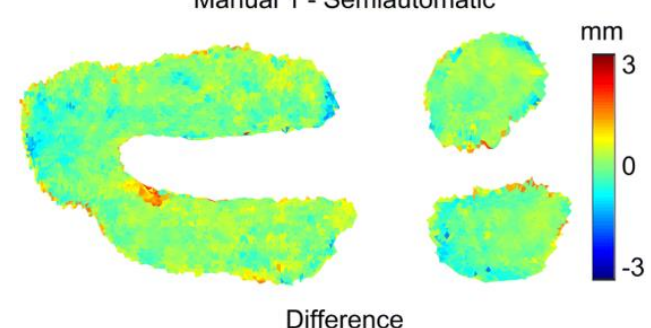

Difference

Manual 2 - Semiautomatic

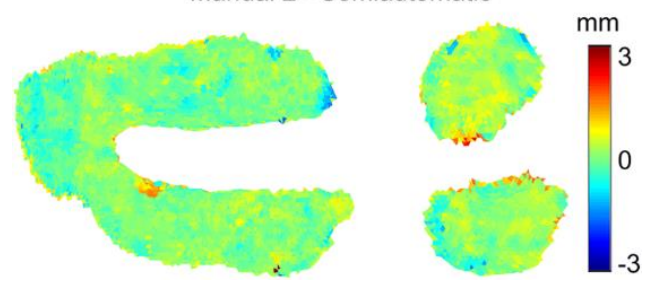

Figure 4. Thicknesses of femoral (first column) and tibial (second column) cartilages for one representative patient and the difference between the thicknesses based on manual and semiautomatic segmentations. Minor differences in determined cartilage thickness can be seen but the overall correspondence is good; the differences are close to zero and the spatial distribution of thickness is similar between all the segmentations. 
The volumes (mean $\pm \mathrm{SD}$ ) of femoral cartilage, determined from CT segmentations, were $7.81 \pm 1.30$, $7.64 \pm 1.30$, and $8.42 \pm 1.08 \mathrm{~cm}^{3}$ when determined using Semiautomatic, Manual 1, and Manual 2 segmentations, respectively. The corresponding values for tibial cartilage were $3.42 \pm 0.44$, $3.29 \pm 0.62$, and $3.43 \pm 0.55 \mathrm{~cm}^{3}$. When comparing the volumes of femoral cartilage, the difference was statistically significant only between the two manual segmentations $(p=0.003)$. No significant differences were found in volume of tibial cartilage between the segmentations.

Manual segmentations based on MR images were compared with the segmentations based on CT images (Table 3 ). The only statistically significant difference $(p=0.020)$ was found in the sensitivity of segmentations of femoral cartilage. Otherwise, no significant difference was found between DSC, sensitivity or specificity values, either in femoral or tibial cartilage; manually and semiautomatically segmented cartilages from CT images corresponded equally well with manual segmentations based on MR images. However, DSC values between MR and CT segmentations (0.67-0.70) were significantly lower $(p<0.029)$ than those between the manual and semiautomatic CT segmentations (0.79-0.83; Table 2). 
Table 3. Manual segmentation from MR images are compared with semiautomatic and manual segmentation from CT images: Dice similarity coefficients, sensitivity and specificity are determined.

\begin{tabular}{clcc}
\hline & \multicolumn{1}{c}{ FEMUR } & MEAN & SD \\
\hline $\begin{array}{c}\text { Dice } \\
\text { Similarity } \\
\text { Coefficient }\end{array}$ & Manual MR vs. Semiautomatic CT & 0.67 & 0.06 \\
& Manual MR vs. Manual CT & 0.70 & 0.06 \\
Sensitivity & Manual MR vs. Semiautomatic CT & 0.55 & 0.07 \\
& Manual MR vs. Manual CT & 0.59 & 0.07 \\
Specificity & Manual MR vs. Semiautomatic CT & 0.90 & 0.02 \\
& Manual MR vs. Manual CT & 0.90 & 0.04 \\
\hline \multirow{2}{*}{ Dice } & Manual MR vs. Semiautomatic CT & 0.69 & 0.03 \\
Similarity & Manual MR vs. Manual CT & 0.70 & 0.04 \\
Coefficient & & & \\
Sensitivity & Manual MR vs. Semiautomatic CT & 0.64 & 0.04 \\
& Manual MR vs. Manual CT & 0.64 & 0.05 \\
Specificity & Manual MR vs. Semiautomatic CT & 0.80 & 0.03 \\
& Manual MR vs. Manual CT & 0.82 & 0.07 \\
\hline
\end{tabular}

.... Satistically (Friedman's test) significantly different $(p<0.05)$ values are connected with a dashed line. 
No significant difference in tibial cartilage volume was found between semiautomatic CT and manual MR segmentation. On the contrary, tibial cartilage volumes determined from manual segmentations of CT images (Manuals 1 and 2) differed statistically significantly ( $p=0.001$ and 0.037 , respectively) from the volumes of manually segmented MR images. Femoral cartilage volumes differed statistically significantly when comparing Manual 1 and Semiautomatic CT segmentations with MR segmentations ( $p<0.001$ and 0.002 , respectively) but no significant difference was detected between Manual 2 and MR segmentations.

The time needed for segmentation was considerably lower when using the semiautomatic method (Supplementary material, Table 1). With femoral cartilage, the average time for manual work was reduced from 290 to $20 \mathrm{~min}$ and with tibial cartilage from 160 to $20 \mathrm{~min}$. The time required for computer processing was only a few minutes.

\section{Discussion}

In this study, a semiautomatic segmentation method for CECT imaging of knee joint was introduced. The correspondence between the reference manual segmentations and segmentations conducted by the proposed method was shown to be good. The method was found feasible to segment both femoral and tibial articular cartilages. Segmenting and analysing trabecular and cortical bone are also feasible since the techniques used are originally developed for this purpose. 41

The DSC, sensitivity, and specificity values were high between semiautomatic and manual CT segmentations. However, the agreement between manual segmentations was slightly better than that between the semiautomatic and manual ones. A partial volume effect limits the accuracy in voxelbased manual segmentations. This might be one of the reasons behind the difference in $D S C$ values between manual and semiautomatic segmentations. In femoral cartilage, the sensitivity between semiautomatic segmentation and one of the manual segmentations was found to be higher than that between the manual ones. The values of these parameters representing the correspondence between segmentations were only slightly, although statistically significantly, different. Currently, the novel, advanced methods 29 for segmenting cartilage from MR images have DSC indices of approximately $0.84-0.87$ being very similar to our results.

The mean cartilage thicknesses and volumes determined using manual and semiautomatic CT segmentations correlated well (Fig. 3). Even though the differences in segmented cartilage thickness were higher at the joint periphery than in the contact area (Fig. 4), the mean absolute differences of 
thickness were around or less than one pixel. Spatial correspondence was good between the segmentation methods. However, some discrepancies were revealed. In the future, these variations could possibly be reduced by using a multi-atlas approach5,48 or statistical shape models 44 to enable a more accurate determination of the cartilage region during the segmentation. Furthermore, the halfmaximum algorithm could be improved; the position of cartilage surface could be determined from the intensity profile, i.e., using nonlinear least squares estimation previously presented for cortical surface estimation.41 However, this point spread function-based cortex estimation is not directly applicable for the estimation of cartilage surface from CECT images due to the diffusion of the contrast agent. Therefore, we decided to use a half-maximum approach where fewer assumptions are needed. Despite the use of only one template, the results are very promising for further development. Furthermore, the number of vertices $\left(0.6 / \mathrm{mm}^{2}\right)$ was found to be adequate for the semiautomatic segmentation.

Manual MR segmentation, as compared with CT segmentations, either manual or semiautomatic, had lower DSC values than when comparing manual CT segmentations with semiautomatic CT segmentations. This may be due to difference in cartilage volumes; volumes based on MR images were systematically higher than those based on CT images. The size of a voxel is eight times greater in MR images compared with CT images, which, unavoidably, increases the partial volume effect leading to greater volumes of segmented cartilage in MR images. Due to its higher resolution, CT images are suggested to allow segmentation of cartilage defects in more detail15 leading possibly to a smaller volume of the defective areas. On the other hand, slightly poorer contrast at the periphery of cartilage might have caused an underestimation of cartilage volume in CT images.

Methods to segment articular cartilage of knee joint have evolved from texture-based methods and now rely on more sophisticated machine learning, atlas-based, and model-based approaches.29 Fripp et al. presented a method to segment cartilages of the knee joint from MR images in which, similarly to the present method, the bones are segmented before the cartilages.10 Both studies indicate that this segmentation strategy seems to achieve a high accuracy. However, the method by Fripp et al. was tested only with cartilages that were intact, i.e., had no pathological changes, whereas our proposed method has been demonstrated to work also with damaged cartilage. Indeed, all of the analysed knees used in this study had clinically diagnosed pathological changes; the knees displayed lesions of ICRS I-III of a severity graded by a clinician (International Cartilage Repair Society grading system). Tabrizi et al. introduced a method for segmenting acetabular cartilage from CECT images,39 however, due to more the complex shape of the knee joint, the present results are not directly 
comparable with those of Tabrizi et al. Machine learning is also a promising approach for knee cartilage segmentation,9,22,49 however, it usually requires a large training set. Generally, previous segmentation methods rely on voxel-based segmentation, possibly leading to inadequate accuracy due to relatively large voxel size or slice thickness with relation to, in some places, very thin cartilage.

Despite the different spatial resolutions between imaging modalities, we compared the CT segmentations with those of MRI. As the MRI is the most widely applied method for clinical imaging of cartilage, and traditionally relies on manual segmentation, it serves well as a reference for the novel method. CT differs from MRI - both methods have their advantages and disadvantages in the diagnostics of OA.20,23,33,40,47 Although MRI is the gold standard for visualizing soft tissues it lacks a clinically acceptable imaging time when very high resolution is needed and, thus, usually has a poorer isotropic resolution than CT in clinical setups. Moreover, the current and conventionally used clinical MRI protocols have a lower contrast in bone, as compared with CT, resulting in a lower sensitivity in detecting bone alterations. As an advantage, CT enables the detection of subtle, OArelated changes in bone.41 However, imaging without contrast enhancement makes it unable to define the morphology of articular cartilage layer. Recently, CECT was shown to enable simultaneous quantitative determination of changes in cartilage and bone in knee joints in vivo.15,26 Utilization of ionizing radiation and the need for an intra-articular injection are drawbacks of CECT; nonetheless, in vivo imaging of knee requires only a small radiation dose,45 i.e., equal to about 2 weeks' background radiation, and the risk of infection due to contrast agent injection is very small.

In contrast-enhanced imaging, the arthrographic CECT images are used for segmentation and dCTa images for analysing the composition of cartilage. In order to analyse the composition of cartilage from dCTa images, co-registering of segmented arthrographic CECT images with dCTa images is required. Thus, the only additional part needed in the analysis is the generation of 3D bone meshes from dCTa images for instance, using Stradwin - the rest of the steps would be automatic. In the future, automated segmentation may be possible directly from dCTa images by utilizing a dual contrast agent technique,32 enabling to omit the acquisition of the arthrographic CECT image. The diffusion of anionic and cationic CT contrast agents has been shown to be related to the tissue's GAG contents, 1,24,25,35,37 thus providing information about the composition of the cartilage. This study utilized an anionic contrast agent; however, cationic agents display a higher sensitivity for GAG content,1,19 suggesting that these could be a good alternative. Furthermore, diffusion is depthdependent, i.e., diffusion is slower in the deep cartilage possibly due to, for instance, the denser collagen network.35 Possibly, in situations where the quality of the cartilage would be very poor, the 
diffusion of contrast agent may already occur during arthrographic CECT and, further, be detected as a thinning of cartilage tissue. In addition, previous in vivo $\mathrm{dCTa}$ studies have reported that the diffusion of the contrast agent is higher close to cartilage lesions.15,26

One advantage of the present study is that the novel method was evaluated using images of patients having knee symptoms, as confirmed by a clinician. However, this imposes a limitation that manual segmentations were the only option for reference since the images were acquired in vivo and, therefore, high-resolution imaging, i.e., $\mu \mathrm{CT}$, or extracting tissue samples to determine exact morphology of the joint, was not possible. Two of the authors segmented all of the knees to minimize this limitation and to make the validation more comprehensive. The mean cartilage thickness contains no information specifically related to lesion detection, but provides information on overall agreement of the segmentation methods. Instead, cartilage thickness maps indicate specifically regions with locally low cartilage thicknesses. Although the present method detects decrease in cartilage thickness, the determination of very sharp-edged or fissure-shaped lesions will need to be further evaluated in the future. Furthermore, even though the present results suggest that the introduced technique is functional, the current number of the patients is relatively low and hence, variations of knee shape and size in general population could not be fully covered.

As another limitation, the presented segmentation method is not fully automatic but requires some user interaction. The semiautomatic segmentations showed statistically insignificant differences between the intra-user and inter-user values for $D S C$, sensitivity and specificity, indicating good repeatability. Furthermore, ICC values for thickness and volume were high. However, the DSC, sensitivity, and specificity values between semiautomatic segmentations were good instead of excellent, indicating that user interaction exert some effect on the segmentation results. The semiautomated bone surface generation was chosen since Stradwin software has been shown to perform adequately for bone cortical surface construction and further enables a quantitative evaluation of both cortical thickness and density in sub-pixel accuracy.41 Since the thickness of both cortical bone and articular cartilage could be very small, the method has a major advantage in accuracy. Furthermore, the geometries of cartilages were determined at the subpixel level conferring a distinct advantage compared with other existing segmentation methods which almost invariably have voxel-based approaches. Additionally, the time for user interaction ( $40 \mathrm{~min})$ is more reasonable whereas the time $(\sim 450 \mathrm{~min})$ needed for fully manual segmentation is unfeasible for clinical use. Furthermore, the computer-aided segmentation part is computationally relatively light and can be undertaken by any regular computer. In contrast, when using some atlas-based methods, 
the algorithms and toolkits seem to rely on excessive computations, thus being unsuitable for realtime applications or requiring GPU calculation.29

In the future, the proposed approach could be used for quantitative analysis of the knee joint, 15,26 especially for the diagnostics of post-traumatic OA, cartilage injuries or to define the stage of OA. When combined with subchondral bone thickness and density analysis, 41 the technique could enable comprehensive and advanced evaluation at an early stage of the disease. Moreover, since the template bone is registered with every segmentation, the presented segmentation method could be used to generate the isotopological mesh needed in statistical shape modelling. 44 This could be advantageous in constructing the biomechanical finite element model of the knee31,46 since the boundary conditions and material properties could be introduced from the template for each corresponding element. The postulated approach would make possible a more rapid implementation of these models since the segmentation is readily in the mesh form that could be used to generate biomechanical models of the knee. Since the accuracy of the semiautomatic segmentations falls between the variability of manual segmentations, which are usually used to generate the models, the devised method can be considered as accurate enough for finite element analysis, a proposal that should be studied in the future. In general, automatic segmentation would be advantageous in several fields in orthopaedics.

As a summary, of our best knowledge, this is the first study to introduce segmentation of knee joint structures from CECT images with minimal user interactions. The contrast enhancement enabled the segmentation of the whole articular cartilage with a time and resource input that is clinically feasible. Furthermore, the segmentations using the proposed method displayed a good correspondence with results gathered by manual segmentation.

\section{Acknowledgments}

The authors acknowledge the Research Committee of the Kuopio University Hospital Catchment Area for the State Research Funding (Projects 5041746, 5041757, and 5203101). Study is also supported by Doctoral Program in Science, Technology and Computing (SCITECO, University of Eastern Finland), Finnish Cultural Foundation, and Academy of Finland (Projects 269315 and 307932). 


\section{Conflict of interest}

Authors declare no conflicts of interest.

\section{Supplementary material}

Supplementary material 1

\section{References}

1.

Bansal, P. N., N. S. Joshi, V. Entezari, B. C. Malone, R. C. Stewart, B. D. Snyder, and M. W. Grinstaff. Cationic contrast agents improve quantification of glycosaminoglycan (GAG) content by contrast enhanced CT imaging of cartilage. J. Orthop. Res. 29:704$709,2011$.

2.

Bookstein, F. L. Principal warps: thin-plate splines and the decomposition of deformations. IEEE Trans. Pattern Anal. Mach. Intell. 11:567-585, 1989.

3.

Buckwalter, J. A., J. L. Marsh, T. Brown, A. Amendola, and J. A. Martin. Articular cartilage injury. In: Principles of Tissue Engineering, edited by R. Lanza, R. Langer, and J. P. Vacanti. Elsevier, 2014, pp. 1253-1266.

4.

Burr, D. B., and M. A. Gallant. Bone remodelling in osteoarthritis. Nat. Rev. Rheumatol. 8:665-673, 2012. 
5.

Chu, C., J. Bai, X. Wu, and G. Zheng. MASCG: Multi-Atlas Segmentation Constrained Graph method for accurate segmentation of hip CT images. Med. Image Anal. 26:173$184,2015$.

6.

Chu, C., C. Chen, L. Liu, and G. Zheng. FACTS: fully automatic CT segmentation of a hip joint. Ann. Biomed. Eng. 43:1247-1259, 2015.

7.

Favre, J., S. F. Scanlan, J. C. Erhart-Hledik, K. Blazek, and T. P. Andriacchi. Patterns of femoral cartilage thickness are different in asymptomatic and osteoarthritic knees and can be used to detect disease-related differences between samples. J. Biomech. Eng. 135:101002, 2013.

8.

Favre, J., J. C. Erhart-Hledik, K. Blazek, B. Fasel, G. E. Gold, and T. P. Andriacchi. Anatomically-standardized maps reveal distinct patterns of cartilage thickness with increasing severity of medial compartment knee osteoarthritis. J. Orthop. Res. 35:2442$2451,2017$.

9.

Folkesson, J., E. B. Dam, O. F. Olsen, P. C. Pettersen, and C. Christiansen. Segmenting articular cartilage automatically using a voxel classification approach. IEEE Trans. Med. Imaging 26:106-115, 2007.

10.

Fripp, J., S. Crozier, S. K. Warfield, and S. Ourselin. Automatic segmentation of articular cartilage in magnetic resonance images of the knee. IEEE Trans. Med. Imaging 29:55-64, 2010.

11. 
Goldring, S. R., and M. B. Goldring. Changes in the osteochondral unit during osteoarthritis: structure, function and cartilage-bone crosstalk. Nat. Rev. Rheumatol. 12:632-644, 2016.

12.

Guermazi, A., D. Hayashi, F. W. Roemer, J. Niu, E. K. Quinn, M. D. Crema, M. C. Nevitt, J. Torner, C. E. Lewis, and D. T. Felson. Partial- and full-thickness focal cartilage defects equally contribute to development of new cartilage damage in knee osteoarthritis - the Multicenter Osteoarthritis Study. Arthritis Rheumatol. 69:560-564, 2016.

13.

Kladny, B., P. Martus, K.-H. Schiwy-Bochat, G. Weseloh, and B. Swoboda. Measurement of cartilage thickness in the human knee-joint by magnetic resonance imaging using a three-dimensional gradient-echo sequence. Int. Orthop. 23:264-267, 1999.

14.

Kokkonen, H. T., J. Mäkelä, K. A. M. Kulmala, L. Rieppo, J. S. Jurvelin, V. Tiitu, H. M. Karjalainen, R. K. Korhonen, V. Kovanen, and J. Töyräs. Computed tomography detects changes in contrast agent diffusion after collagen cross-linking typical to natural aging of articular cartilage. Osteoarthr. Cartil. 19:1190-1198, 2011.

15.

Kokkonen, H. T., J. Suomalainen, A. Joukainen, H. Kröger, J. Sirola, J. Jurvelin, J. Salo, and J. Töyräs. In vivo diagnostics of human knee cartilage lesions using delayed CBCT arthrography. J. Orthop. Res. 32:403-412, 2014.

16.

Kotlarz, H., C. L. Gunnarsson, H. Fang, and J. A. Rizzo. Insurer and out-of-pocket costs of osteoarthritis in the US: evidence from national survey data. Arthritis Rheumatol. 60:3546-3553, 2009.

17. 
Kroon, D. J. Finite Iterative Closest Point. Natick: Mathworks, 2009.

18.

Kulmala, K. A. M., H. M. Karjalainen, H. T. Kokkonen, V. Tiitu, V. Kovanen, M. J. Lammi, J. S. Jurvelin, R. K. Korhonen, and J. Töyräs. Diffusion of ionic and non-ionic contrast agents in articular cartilage with increased cross-linking-contribution of steric and electrostatic effects. Med. Eng. Phys. 35:1415-1420, 2013.

19.

Lakin, B. A., H. Patel, C. Holland, J. D. Freedman, J. S. Shelofsky, B. D. Snyder, K. S. Stok, and M. W. Grinstaff. Contrast-enhanced CT using a cationic contrast agent enables non-destructive assessment of the biochemical and biomechanical properties of mouse tibial plateau cartilage. J. Orthop. Res. 34:1130-1138, 2016.

20.

Lakin, B. A., B. D. Snyder, and M. W. Grinstaff. Assessing cartilage biomechanical properties: techniques for evaluating the functional performance of cartilage in health and disease. Annu. Rev. Biomed. Eng. 19:27-55, 2017.

21.

Li, H., R. W. Sumner, and M. Pauly. Global correspondence optimization for non-rigid registration of depth scans. Eurograph. Symp. Geom. Process. 27:1421-1430, 2008.

22.

Liu, F., Z. Zhou, H. Jang, A. Samsonov, G. Zhao, and R. Kijowski. Deep convolutional neural network and 3D deformable approach for tissue segmentation in musculoskeletal magnetic resonance imaging. Magn. Reson. Med. 79:2379-2391, 2018.

23.

Matzat, S. J., F. Kogan, G. W. Fong, and G. E. Gold. Imaging strategies for assessing cartilage composition in osteoarthritis. Curr. Rheumatol. Rep. 16:462, 2015. 
24.

Mittelstaedt, D., and Y. Xia. Depth-dependent glycosaminoglycan concentration in articular cartilage by quantitative contrast-enhanced micro-computed tomography. Cartilage 6:216-225, 2015.

25.

Moshtagh, P. R. R., B. Pouran, J. van Tiel, J. Rauker, M. R. R. Zuiddam, V. Arbabi, N. M. M. Korthagen, H. Weinans, and A. A. A. Zadpoor. Micro- and nano-mechanics of osteoarthritic cartilage: the effects of tonicity and disease severity. J. Mech. Behav. Biomed. Mater. 59:561-571, 2016.

26.

Myller, K. A. H., M. J. Turunen, J. T. J. Honkanen, S. P. Väänänen, J. T. Iivarinen, J. Salo, J. S. Jurvelin, and J. Töyräs. In vivo contrast-enhanced cone beam CT provides quantitative information on articular cartilage and subchondral bone. Ann. Biomed. Eng. 45:811-818, 2017.

27.

Olson, S. A., B. D. Furman, V. B. Kraus, J. L. Huebner, and F. Guilak. Therapeutic opportunities to prevent post-traumatic arthritis: lessons from the natural history of arthritis after articular fracture. J. Orthop. Res. 33:1266-1277, 2015.

28.

Pedoia, V., X. Li, F. Su, N. Calixto, and S. Majumdar. Fully automatic analysis of the knee articular cartilage T1p relaxation time using voxel-based relaxometry. J. Magn. Reson. Imaging 43:970-980, 2016.

29.

Pedoia, V., S. Majumdar, and T. M. Link. Segmentation of joint and musculoskeletal tissue in the study of arthritis. Magn. Reson. Mater. Phys. 29:207-221, 2016. 
Pereanez, M., K. Lekadir, I. Castro-Mateos, J. M. Pozo, A. Lazary, and A. F. Frangi. Accurate segmentation of vertebral bodies and processes using statistical shape decomposition and conditional models. IEEE Trans. Med. Imaging 34:1627-1639, 2015.

31.

Pierce, D. M., W. Trobin, S. Trattnig, H. Bischof, and G. A. Holzapfel. A phenomenological approach toward patient-specific computational modeling of articular cartilage including collagen fiber tracking. J. Biomech. Eng. 131:91006, 2009.

32.

Saukko, A. E. A., J. T. J. Honkanen, W. Xu, S. P. Väänänen, J. S. Jurvelin, V.-P. Lehto, and J. Töyräs. Dual contrast CT method enables diagnostics of cartilage injuries and degeneration using a single CT image. Ann. Biomed. Eng. 45:2857-2866, 2017.

33.

Shafieyan, Y., N. Khosravi, M. Moeini, and T. M. Quinn. Diffusion of MRI and CT contrast agents in articular cartilage under static compression. Biophys. J. 107:485-492, 2014.

34.

Shim, H., S. Chang, C. Tao, J.-H. Wang, C. Kent Kwoh, and K. T. Bae. Knee cartilage: efficient and reproducible segmentation on high-spatial-resolution MR images with the semiautomated graph-cut algorithm method. Radiology 251:548-556, 2009.

35.

Silvast, T. S., J. S. Jurvelin, M. J. Lammi, and J. Töyräs. pQCT study on diffusion and equilibrium distribution of iodinated anionic contrast agent in human articular cartilageassociations to matrix composition and integrity. Osteoarthr. Cartil. 17:26-32, 2009.

36.

Sölveborn, S. A. Trauma. In: Emergency Orthopedics: A Manual on Acute Conditions of the Locomotor System, edited by S. A. Sölveborn. Berlin: Springer, 2014, pp. 67-70. 
37.

Stewart, R. C., J. T. J. Honkanen, H. T. Kokkonen, V. Tiitu, S. Saarakkala, A. Joukainen, B. D. Snyder, J. S. Jurvelin, M. W. Grinstaff, and J. Töyräs. Contrast-enhanced computed tomography enables quantitative evaluation of tissue properties at intrajoint regions in cadaveric knee cartilage. Cartilage 8:391-399, 2017.

38.

Tabrizi, P. R., R. A. Zoroofi, F. Yokota, S. Tamura, T. Nishii, and Y. Sato. Acetabular cartilage segmentation in CT arthrography based on a bone-normalized probabilistic atlas. Int. J. Comput. Assist. Radiol. Surg. 10:433-446, 2015.

39.

Tabrizi, P. R., R. A. Zoroofi, F. Yokota, T. Nishii, and Y. Sato. Shape-based acetabular cartilage segmentation: application to CT and MRI datasets. Int. J. Comput. Assist. Radiol. Surg. 11:1-19, 2016.

40.

Taylor, C., J. Carballido-Gamio, S. Majumdar, and X. Li. Comparison of quantitative imaging of cartilage for osteoarthritis: T2, T1 $\rho$, dGEMRIC and contrast-enhanced computed tomography. Magn. Reson. Imaging 27:779-784, 2009.

41.

Treece, G. M. M., and A. H. H. Gee. Independent measurement of femoral cortical thickness and cortical bone density using clinical CT. Med. Image Anal. 20:249-264, 2015.

42.

Turunen, M. J., J. Töyräs, H. T. Kokkonen, and J. S. Jurvelin. Quantitative evaluation of knee subchondral bone mineral density using cone beam computed tomography. IEEE Trans. Med. Imaging 34:2186-2190, 2015.

43. 
Väänänen, S. P., J. S. Jurvelin, and H. Isaksson. Estimation of 3-D shape, internal density and mechanics of proximal femur by combining bone mineral density images with shape and density templates. Biomech. Model. Mechanobiol. 11:791-800, 2012.

44.

Väänänen, S. P., L. Grassi, G. Flivik, J. S. Jurvelin, and H. Isaksson. Generation of 3D shape, density, cortical thickness and finite element mesh of proximal femur from a DXA image. Med. Image Anal. 24:125-134, 2015.

45.

van Tiel, J., M. Siebelt, J. H. Waarsing, T. M. Piscaer, M. van Straten, R. Booij, M. L. Dijkshoorn, G. J. Kleinrensink, J. A. N. Verhaar, G. P. Krestin, H. Weinans, and E. H. G. Oei. CT arthrography of the human knee to measure cartilage quality with low radiation dose. Osteoarthr. Cartil. 20:678-685, 2012.

46.

Venäläinen, M. S., M. E. Mononen, J. Salo, L. P. Räsänen, J. S. Jurvelin, J. Töyräs, T. Virén, and R. K. Korhonen. Quantitative evaluation of the mechanical risks caused by focal cartilage defects in the knee. Sci. Rep. 6:37538, 2016.

47.

Wang, Y., A. J. Teichtahl, and F. M. Cicuttini. Osteoarthritis year in review 2015: imaging. Osteoarthr. Cartil. 24:49-57, 2016.

48.

Xia, Y., J. Fripp, S. S. Chandra, R. Schwarz, C. Engstrom, and S. Crozier. Automated bone segmentation from large field of view 3D MR images of the hip joint. Phys. Med. Biol. 58:7375-7390, 2013.

49. 
Yin, Y., X. Zhang, R. Williams, X. Wu, D. D. Anderson, and M. Sonka. LOGISMOSlayered optimal graph image segmentation of multiple objects and surfaces: cartilage segmentation in the knee joint. IEEE Trans. Med. Imaging 29:2023-2037, 2010.

50.

Zhang, K., W. Lu, and P. Marziliano. Automatic knee cartilage segmentation from multicontrast MR images using support vector machine classification with spatial dependencies. Magn. Reson. Imaging 31:1731-1743, 2013.

51.

Zou, K. H., S. K. Warfield, A. Bharatha, C. M. C. Tempany, M. R. Kaus, S. J. Haker, W. M. Wells, F. A. Jolesz, and R. Kikinis. Statistical validation of image segmentation quality based on a spatial overlap index. Acad. Radiol. 11:178-189, 2004. 